University of Wollongong

Research Online

Faculty of Informatics - Papers (Archive)

Faculty of Engineering and Information

Sciences

$1-1-2011$

\title{
Partially overlapped range images registration based on fringe pattern profilometry
}

Xiaochen $\mathrm{He}$

University of Wollongong, xiahe@uow.edu.au

Jiangtao Xi

University of Wollongong, jiangtao@uow.edu.au

Yanguang Yu

University of Wollongong, yanguang@uow.edu.au

Follow this and additional works at: https://ro.uow.edu.au/infopapers

Part of the Physical Sciences and Mathematics Commons

\section{Recommended Citation}

$\mathrm{He}$, Xiaochen; Xi, Jiangtao; and Yu, Yanguang: Partially overlapped range images registration based on fringe pattern profilometry 2011.

https://ro.uow.edu.au/infopapers/1992

Research Online is the open access institutional repository for the University of Wollongong. For further information contact the UOW Library: research-pubs@uow.edu.au 


\title{
Partially overlapped range images registration based on fringe pattern profilometry
}

\author{
Abstract \\ Registration of two three-dimensional (3-D) point sets is a fundamental problem of 3-D shape \\ measurement and modeling pipeline. This paper investigates the automatic pair-wise method to register \\ partially overlapped range images generated by self-developed fringe pattern profilometry (FPP) system. \\ The method is based on the classic iterative closest point (ICP) algorithm but combined with several \\ extensions to adapt to the experimental data. Firstly, the distance function for correspondence finding is \\ modified to be the weighted linear combination of positions and Euclidean invariant features for \\ improving the probability of convergence. In addition, outliers can be discarded through robust statistics \\ and adaptive thresholding of weighted distances between corresponding point pairs. Both artificial and \\ real data are used to test the proposed method. In the ideal noise-free conditions, the experimental \\ results illustrate that it converges to the global minima. The experimental results also show that the \\ proposed method increases the possibility of global convergence when deal with partially overlapped \\ range images.
}

\section{Keywords}

partially, registration, images, fringe, range, overlapped, pattern, profilometry

\section{Disciplines}

Physical Sciences and Mathematics

\section{Publication Details}

$\mathrm{He}, \mathrm{X} ., \mathrm{Xi}, \mathrm{J}$. \& Yu, Y. (2011). Partially overlapped range images registration based on fringe pattern profilometry. 2011 International Conference on Optical Instruments and Technology: Optoelectronic Imaging and Processing Technology (pp. 8200F-1-82000F-12). USA: SPIE. 


\title{
Partially Overlapped Range Images Registration Based on Fringe Pattern Profilometry
}

\author{
Xiaochen $\mathrm{He}^{*^{\mathrm{a}}}$, Jiangtao $\mathrm{Xi}^{\mathrm{a}}$, Yanguang $\mathrm{Yu}^{\mathrm{a}}$ \\ ${ }^{a}$ School of Electrical, Computer and Telecommunications Engineering, University of Wollongong, \\ Northfields Ave, Wollongong, NSW, 2522, Australia \\ *Email: jiangtao@uow.edu.au; Phone: +61 4221 3412;
}

\begin{abstract}
Registration of two three-dimensional (3-D) point sets is a fundamental problem of 3-D shape measurement and modeling pipeline. This paper investigates the automatic pair-wise method to register partially overlapped range images generated by self-developed fringe pattern profilometry (FPP) system. The method is based on the classic iterative closest point (ICP) algorithm but combined with several extensions to adapt to the experimental data. Firstly, the distance function for correspondence finding is modified to be the weighted linear combination of positions and Euclidean invariant features for improving the probability of convergence. In addition, outliers can be discarded through robust statistics and adaptive thresholding of weighted distances between corresponding point pairs. Both artificial and real data are used to test the proposed method. In the ideal noise-free conditions, the experimental results illustrate that it converges to the global minima. The experimental results also show that the proposed method increases the possibility of global convergence when deal with partially overlapped range images.
\end{abstract}

Keywords: Range image registration, iterative closest point, invariant feature, fringe pattern profilometry

\section{INTRODUCTION}

Three-dimensional (3-D) measurement techniques have emerged for several years and have been applied in practice such as the quality inspection in the reverse engineering, rapidly prototyping in the manufacture industry, aesthetic restoration and reconstruction in medical and archaeology, virtual reality for entertainment.

Typically, two stages are involved during the 3-D measurement: data acquisition, and data visualization. Data acquisition is the process of obtaining data of the sampling points on the surface ${ }^{[1]}$. A vast of 3-D shape acquisition methods have evolved over the past years. The current tendency evolves towards the high-density non-contact optical measurement with the rapid development of the related hardware. Fringe pattern profilometry (FPP) ${ }^{[2]}$ is one of most promising technique due to its fast, low-cost, and potentially high accuracy.

Data visualization is a processing of of computer modeling by using the acquisition data. Universal commercial software platforms have been developed for data visualization. Nowadays, the combination of data acquisition techniques with universal commercial software platform is a popular solution of 3-D metrology. However either the existing commercial 3-D measurement technique or the universal software platform is very expensive. This paper provides an implementation of 3-D shape measurement using a self-developed FPP system and also the basic knowledge about modeling.

Registration of two 3-D point sets is a fundamental problem of shape acquisition and modeling pipeline. It aims to find a set of rigid transformations between two point sets of a given 3-D object taken from different views and align them into a common reference coordinate frame without any assumptions about the object motions ${ }^{[3]}$. Registration works as matching the corresponding point pairs (the same point measured in different views) and estimating the transformations using correspondences. The existing registration methods can be roughly categorized as coarse registration and fine registration according to whether the initial estimation is provided. Among the fine registration methods, one of the most popular one is the iterative closest point (ICP) algorithm. Since the first appearance of ICP, various algorithms based on ICP have been proposed and summarized in ${ }^{[8]}$. The main issues of ICP variants are finding correspondences and estimating transformation between two point sets. This kind of method seeks the corresponding points of one point set in the other point set by using the Euclidean closest distance and estimate rigid motion that best align the point sets by using Singular Value Decomposition (SVD) ${ }^{[5]}$, Unit Quaternion (UQ) ${ }^{[6]}$ or Dual Quaternion (DQ) ${ }^{[7]}$ iteratively until the mean squared of the distances between all corresponding point pairs converges to a global minimum. Chen and Medioni

2011 International Conference on Optical Instruments and Technology: Optoelectronic Imaging and Processing Technology, edited by Toru Yoshizawa, Ping Wei, Jesse Zheng, Proc. of SPIE Vol. 8200, 82000F · () 2011 SPIE · CCC code:

0277-786X/11/\$18 doi: 10.1117/12.906556

Proc. of SPIE Vol. $820082000 \mathrm{~F}-1$ 
develop a similar method with Besl and McKay's but using different distance criteria. Sharp et al. use Euclidean invariant features in conjunction with positions to choose point correspondences for improving convergence ${ }^{[9]}$. Zhang extends ICP to reject some point pairs whose distances are larger than the adaptive threshold obtained by analyzing the statistics of all distances rather than exploiting all distances to estimate transformation, and improve the robustness to outliers and noise ${ }^{[10]}$. In addition, many other researchers contribute to improve the robustness of ICP, such as the picky ICP proposed by Zinsser et al. ${ }^{[11]}$, RICP proposed by Trucco ${ }^{[12]}$ and MICP proposed by Masuda and Yokoya ${ }^{[13]}$. In general, ICP-based methods give the relatively accurate estimation of the transformations between different views on condition that a good initial estimation is available.

However, a large number of iterations is required when the initial guess is not optimal, especially the registration of partially overlapping point sets. The proposed method combines Euclidean invariant features with the modified ICP that merges several existing extensions of ICP, which improves the precision and reduces the iteration times in the partially overlapped registration. In the following sections, the background theory about FPP system and the ICP algorithm will be reviewed briefly in the section 2 . The proposed methods are explained in details in the section 3 . In the section 4 , the performance of the proposed method is compared to the baseline method. The section 5 highlights the contributions and draws the conclusions.

\section{BACKGROUND}

\subsection{Fringe Pattern Profilometry (FPP)}

Fringe Pattern Profilometry (FPP) is one of most promising technology for non-contact 3-D shape measurement. One of the most outstanding advantage of FPP is the ability to provide full field-of-view 3-D information with high accuracy. Currently, the commercial FPP system has been applied into the fields like quality inspection in manufacture, terrain exploration in vehicle navigation, art conservation and restoration, precision aid in medical surgery. The 3-D shape measurement by FPP includes digital fringe pattern projection and acquisition, and the 3-D information extraction based on fringe pattern analysis.

\section{Fringe Pattern Projection and Acquisition}

Typically, FPP system consists of a CCD camera, a digital video projector and a computer. The principle of FPP system is described by the schematic systematic diagram shown in the Fig. 1(a). Firstly, one image with special designed structured light patterns is generated by the computer and then projected onto the surface of a reference plane. The reflected intensity image is captured by the CCD camera (e.g., Fig. 1 (b)), denoted as . Then, by removing the reference plane, the same fringe pattern is projected onto the object surface of interest, and another reflected image is captured (e.g., Fig. 1 (c)), which is a deformed version of the reflected image of reference plane due to that the variance of the object surface leads to phase shift of the fringe patterns, denoted as

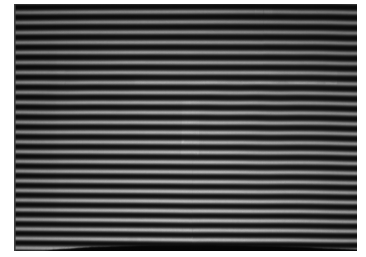

(b)

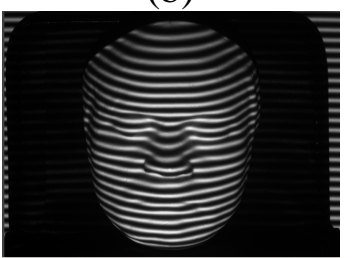

(c)

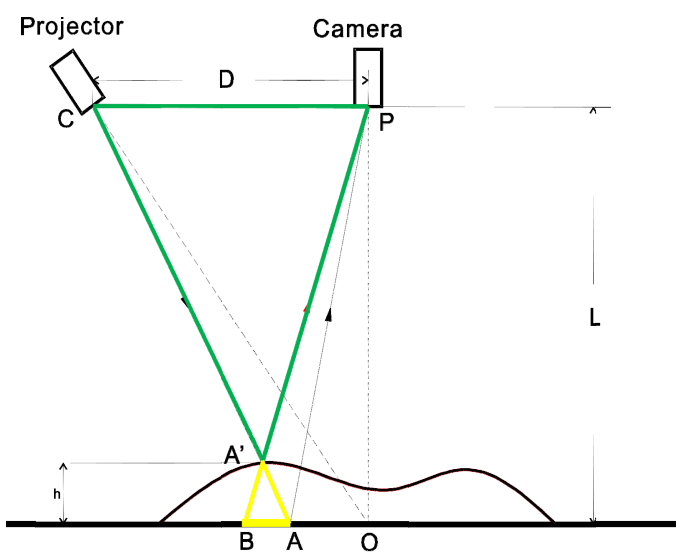

(a)

Fig. 1. (a) the schematic diagram of FPP system (b) fringe pattern projected on reference plane (c) the same fringe pattern projected on the object of interest 
In order to acquire the object surface shape, we can take advantages of the difference between $\mathrm{I}_{\mathrm{r}}(\mathrm{x}, \mathrm{y})$ and $\mathrm{I}_{\mathrm{o}}(\mathrm{x}, \mathrm{y})$ to extract the height distribution. To establish the relationship between $\mathrm{I}_{\mathrm{r}}(\mathrm{x}, \mathrm{y})$ and $\mathrm{I}_{\mathrm{o}}(\mathrm{x}, \mathrm{y})$, one light beam is considered in the Fig. 1(a), which is along the direction of $\overrightarrow{\mathrm{CA}} . \overrightarrow{\mathrm{CPA}}$ is the light route when this beam projected on the reference plane and reflected back to the camera, while $\overrightarrow{\mathrm{CPA}^{\prime}}$ is the light route of this beam when the object exists. It can be observed that the intensity of point $A$ in $\mathrm{I}_{\mathrm{r}}(\mathrm{x}, \mathrm{y})$ and $\mathrm{A}^{\prime}$ in $\mathrm{I}_{\mathrm{o}}(\mathrm{x}, \mathrm{y})$ generated by twice projections along the same direction on reference and on object respectively is the same, which can be expressed as (1). In the following expressions, only one cross section of the object surface for a given $\mathrm{y}$ coordinate is considered so that all the functions are only about $\mathrm{x}$ variable for simplicity.

$$
\mathrm{I}_{\mathrm{r}}\left(\mathrm{x}_{\mathrm{A}}\right)=\mathrm{I}_{\mathrm{o}}\left(\mathrm{x}_{\mathrm{A}^{\prime}}\right)
$$

Furthermore, the position of point $B$ in $I_{r}(x, y)$ and $A^{\prime}$ in $I_{0}(x, y)$ captured by camera is along the same direction. So $\mathrm{x}_{\mathrm{B}}=\mathrm{x}_{\mathrm{A}^{\prime}}$. Let $\mathrm{u}\left(\mathrm{x}_{\mathrm{A}^{\prime}}\right)$ denotes the distance between point $\mathrm{A}$ and $\mathrm{B}$. Therefore, the following equations could be obtained:

$$
\begin{gathered}
\mathrm{I}_{\mathrm{r}}\left(\mathrm{x}_{\mathrm{A}}\right)=\mathrm{I}_{\mathrm{o}}\left(\mathrm{x}_{\mathrm{A}^{\prime}}\right)=\mathrm{I}_{\mathrm{o}}\left(\mathrm{u}\left(\mathrm{x}_{\mathrm{A}^{\prime}}\right)-\mathrm{x}_{\mathrm{A}}\right. \\
\mathrm{u}\left(\mathrm{x}_{\mathrm{A}^{\prime}}\right)=\mathrm{x}_{\mathrm{A}}-\mathrm{x}_{\mathrm{B}}=\mathrm{x}_{\mathrm{A}}-\mathrm{x}_{\mathrm{A}^{\prime}} \Rightarrow \mathrm{x}_{\mathrm{A}^{\prime}}=\mathrm{u}\left(\mathrm{x}_{\mathrm{A}^{\prime}}\right)-\mathrm{x}_{\mathrm{A}}
\end{gathered}
$$

According to the triangulation of FPP system, the height of point $\mathrm{A}^{\prime}$ can be calculated through the equation (4):

$$
\frac{\mathrm{h}\left(\mathrm{x}_{\mathrm{A}^{\prime}}\right)}{\mathrm{L}-\mathrm{h}\left(\mathrm{x}_{\mathrm{A}^{\prime}}\right)}=\frac{\mathrm{u}\left(\mathrm{x}_{\mathrm{A}^{\prime}}\right)}{\mathrm{D}} \Rightarrow \mathrm{h}\left(\mathrm{x}_{\mathrm{A}^{\prime}}\right)=\frac{\mathrm{L} \cdot \mathrm{u}\left(\mathrm{x}_{\mathrm{A}^{\prime}}\right)}{\mathrm{D}+\mathrm{u}\left(\mathrm{x}_{\mathrm{A}^{\prime}}\right)}
$$

where $\mathrm{L}$ is the distance between camera and the reference plane, $\mathrm{D}$ is the distance between the camera and the projector.

\section{Fringe Pattern Analysis for Shape Extraction Based on Phase Detection}

The main task of fringe pattern analysis is to detect $\mathrm{u}(\mathrm{x})$ for height information. There are various algorithms for shape extraction. Among them, Phase Shifting Profilometry (PSP) ${ }^{[2]}$ is one of the most widely algorithm, which is based on phase detection. The principle of PSP is to project multiple periodic fringe patterns, which are generated by shifting the phase of original ideal sinusoidal pattern with $1 / \mathrm{M}, 2 / \mathrm{M}, 3 / \mathrm{M}, \ldots,(\mathrm{M}-1) / \mathrm{M}$ delay, on the reference plane and the object of interest respectively. In the generalized model of M-steps PSP algorithm, the captured phase shifted fringe patterns on the reference plane and on the object surfaces can be expressed respectively as follows:

$$
\begin{aligned}
& \mathrm{I}_{\mathrm{i}}^{\mathrm{r}}(\mathrm{x})=\cos \left(2 \pi f_{o} x+\phi_{0}(\mathrm{x})+\frac{2 \pi(\mathrm{i}-1)}{\mathrm{M}}\right), \text { for } \mathrm{i}=1,2,3, \ldots, \mathrm{M} \\
& \mathrm{I}_{\mathrm{i}}^{\mathrm{o}}(\mathrm{x})=\cos \left(2 \pi f_{o} x+\phi_{1}(\mathrm{x})+\frac{2 \pi(\mathrm{i}-1)}{\mathrm{M}}\right), \text { for } \mathrm{i}=1,2,3, \ldots, \mathrm{M}
\end{aligned}
$$

where $f_{0}$ is the width of each individual fringe on the fringe pattern. $\phi_{0}(\mathrm{x})$ and $\phi_{1}(\mathrm{x})$ can be solved by ${ }^{[2]}$. Moreover, the phase difference $\Delta \varphi(\mathrm{x})$ and $u(x)$ have the relationship as follows:

$$
\varphi(x)=\phi_{1}(\mathrm{x})-\phi_{0}(\mathrm{x})=2 \pi f_{o} u(x)
$$

Therefore, the height of any point on the surface of the object like $\mathrm{A}^{\prime}$ can be retrieved when $\mathrm{D}, \mathrm{L}$ and the phase difference $\Delta \varphi$ are known, which can be expressed as follows:

$$
\mathrm{h}(\mathrm{x})=\frac{\Delta \varphi(\mathrm{x}) \cdot \mathrm{L}}{\Delta \varphi(\mathrm{x})-2 \pi f_{0} \mathrm{D}}
$$

where $\Delta \varphi(\mathrm{x})=\phi_{1}(\mathrm{x})-\phi_{0}(\mathrm{x}), \phi_{0}$ and $\phi_{1}$ are the phases of the fundamental components of $\mathrm{I}_{\mathrm{o}}$ and $\mathrm{I}_{\mathrm{r}}$ respectively.

Fig. 2 shows twelve images captured by the self-developed FPP system. The top six images is a set of fringe patterns with $\pi / 3$ phase stepping projected on the reference and captured by the camera. They can be expressed by (5) while $M=6$. The bottom six images are captured when the same set of fringe patterns are projected on the object (e.g. a plaster Beethoven face), which can be described by (6) while $M=6$. According to the above method for phase detection and phase unwrapping algorithms, a continuous phase difference $\Delta \varphi(\mathrm{x}, \mathrm{y})$ can be obtained. Afterwards, the height map could be obtained through (8) for the 3-D shape reconstruction. 

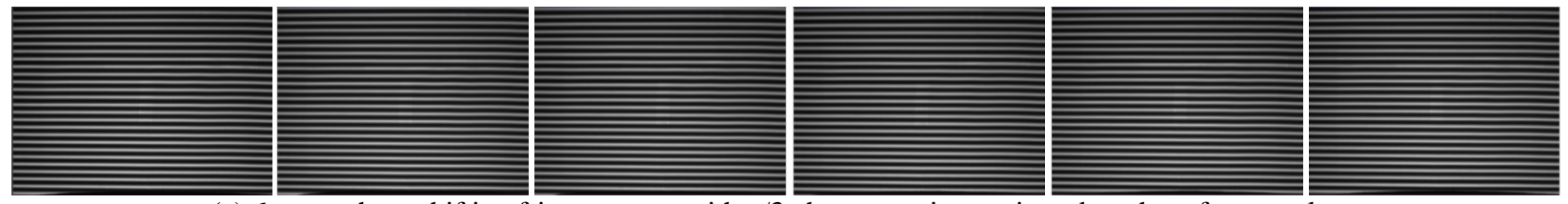

(a) 6-steps phase shifting fringe pattern with $\pi / 3$ phase stepping projected on the reference plane
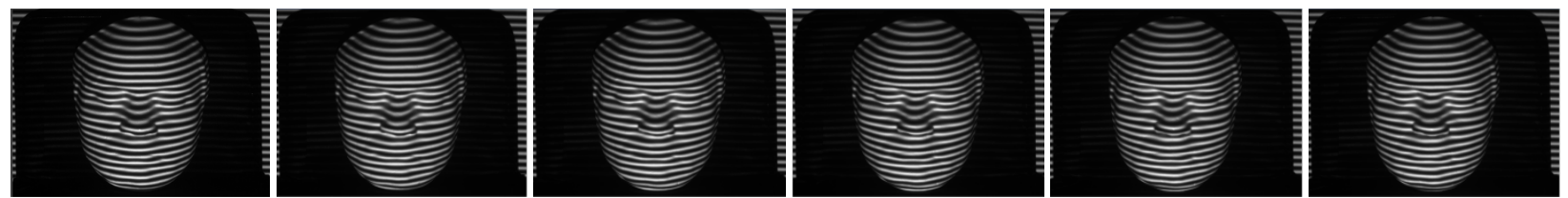

(b) 6-steps phase shifting fringe pattern with $\pi / 3$ phase stepping projected on the object

Fig. 2. Twelve images captured for 6-steps PSP

PSP has the high reputation of high accuracy. The more steps of fringe patterns are used, the more accurate result is obtained, and the higher computation burden must be suffered. In practice, 3-steps PSP is the minimum number of fringe patterns for the shape extraction. Furthermore, the nature of PSP algorithm can reduce the influence from the reflectivity of the object, the sensitivity of camera and ambient light ${ }^{[17]}$. Therefore, we choose 6 steps PSP for high accuracy in order to minimize the measurement error and provide good initial state for the registration.

\section{Post-processing}

FPP generates the height information of the object surface related to the reference plane, which can be organized as height map (i.e. depth map). After calibration of FPP system, the height map can be converted to the range image whose element denotes the distances from the surface points of object to the camera along rays transmitting from a regularly spaced grid within the field of view. In other words, range image is a form of 3-D data representation to describe the sampled point-based 3-D shape but organized as an $\mathrm{M} \times \mathrm{N}$ grid, of which the horizontal and vertical coordinates are implicitly determined by the indices of the grid. The nature of range image maintains the topology and connectivity of measured points on the surface of the object.

\subsection{Iterative closest point (ICP)}

When measuring free-form 3-D shapes or large surface by FPP, it is indispensable to capture a group of range images from multiple successive views (move camera or the object of interest, they are inversely equivalent) that contain enough surface information for modeling the entire 3-D object. Each range image from different views has its own coordinate frame. If the poses of views or the motions of object are hard to record, the fundamental technique that aligns a group of range images from different views and then merging them into an integrated 3-D model is necessary, which is called registration.

Assume $I=\left\{I_{1}, I_{2}, \ldots, I_{n}\right\}$ be a set of range images taken from different viewing directions. Each one has its own coordinate frame. The challenge of registration is to evaluate the Euclidean rigid transformations including the translation $T=\left\{T_{1}, T_{2}, \ldots, T_{n}\right\}$ and rotation vectors $R=\left\{R_{1}, R_{2}, \ldots, R_{n}\right\}$ between a set of range surfaces originated from $I$ and represent them all with respect to a common coordinate frame. For each range image $I_{i} \in I$, the transformation vector $T_{i}$ and $R_{i}$ transforms every point and results $I_{i}^{\prime}$ in the global coordinate frame. The operation of estimating the rigid transformations between inter-frame range images is known as registration, the result of this estimation is called the registration transformation ${ }^{[3]}$.

When a set of range images being registered, the coordinate frame of the first range image is often regarded as the target frame, and the other range images to be aligned to the target frame are called the model frame. If the target frame $\mathrm{P}=\left\{\mathrm{p}_{1}\right.$, $\left.\mathrm{p}_{2}, \ldots, \mathrm{p}_{\mathrm{m}}\right\}$ where $\mathrm{p}_{\mathrm{i}} \mathrm{R}^{3}(\mathrm{i}=1,2, \ldots, \mathrm{m})$ and model frame $\mathrm{Q}=\left\{\mathrm{q}_{1}, \mathrm{q}_{2}, \ldots, \mathrm{q}_{\mathrm{n}}\right\}$ where $\mathrm{q}_{\mathrm{i}} \mathrm{R}^{3}(\mathrm{i}=1,2, \ldots, \mathrm{n})$ are to be registered, the objective of registration is to find a rotation matrix $R$ and a translate vector $T$ which makes the MSE of distances $e$ is minimal,

$$
e=\frac{1}{\mathrm{~N}} \sum_{\mathrm{i}=1}^{\mathrm{N}} \operatorname{dist}\left(\mathrm{Rq}_{\mathrm{i}}+\mathrm{T}, \mathrm{p}_{\mathrm{i}}\right)
$$

where $\mathrm{q}_{\mathrm{i}} \in \mathrm{Q}$ is the point in the overlapping region of the two range images and the dist function calculates the Euclidean distance from $\mathrm{q}_{\mathrm{i}}$ to its corresponding point $\mathrm{p}_{\mathrm{i}} \in \mathrm{P}$. 


\subsection{Local surface descriptors}

The traditional ICP method only takes account of the spatial positions for correspondence searching. Currently, some researchers use surface descriptors to provide additional information for the improvement of correspondence searching. The surface descriptor refers to the geometric description of the local surface features, which provides the attribute of each point and its neighborhood. The most crucial requirement for the surface descriptors is that they must be invariant in the Euclidean space. In other words, the surface descriptors rely on the surface variance but not the Euclidean spatial position. Currently, a variety of descriptors have been proposed such as the differential quantities, integral quantities and some others inspired from the previous two ${ }^{[9,18-21]}$. Among them, the surface curvature is a sort of differential geometric description of local surface features. Curvature reflects the local property of surface, and has no relationship with the parameters and the representation of the surface. Moreover, curvature is the Euclidean invariant of the 3-D rigid transformation. The principle curvatures are commonly used surface descriptors. For every point on a surface, the principle curvatures is two directions with maximum and minimum curvature for all curves on the surface that passes through that point and have the identical normal at that point. The computation of principle curvatures can be referred to [18].

\subsection{Transformation estimation}

Suppose that the coordinates of a number of points in two different Cartesian coordinate systems are given. The problem of recovering the transformation between the two systems from these measurements is referred to as that of absolute orientation. The transformation between two Cartesian coordinate systems can be thought of as the result of a rigid-body motion and can thus be decomposed into a rotation and a translation.

As I mentioned, the successive range images about a 3-D object measured by FPP can be regarded as a number of points measured in different Cartesian coordinate systems. After finding the correspondence information, the next stage in the ICP pipeline is the transformation estimation between Cartesian coordinate systems. Unit quaternion (UQ) ${ }^{[6]}$ and dual quaternion (DQ) ${ }^{[7]}$ algorithm can be used to compute the rigid transformation between two coordinate systems through the use of corresponded point pairs.

\section{PROPOSED METHOD}

My proposed method belongs to the variant of ICP. The main idea is to minimize the mean squared error (i.e. distance between the selected corresponding point pairs iteratively). In the section of 2.2, the principle of ICP method is explained. In general, the pipeline of ICP method can be concluded in the following flow chart:

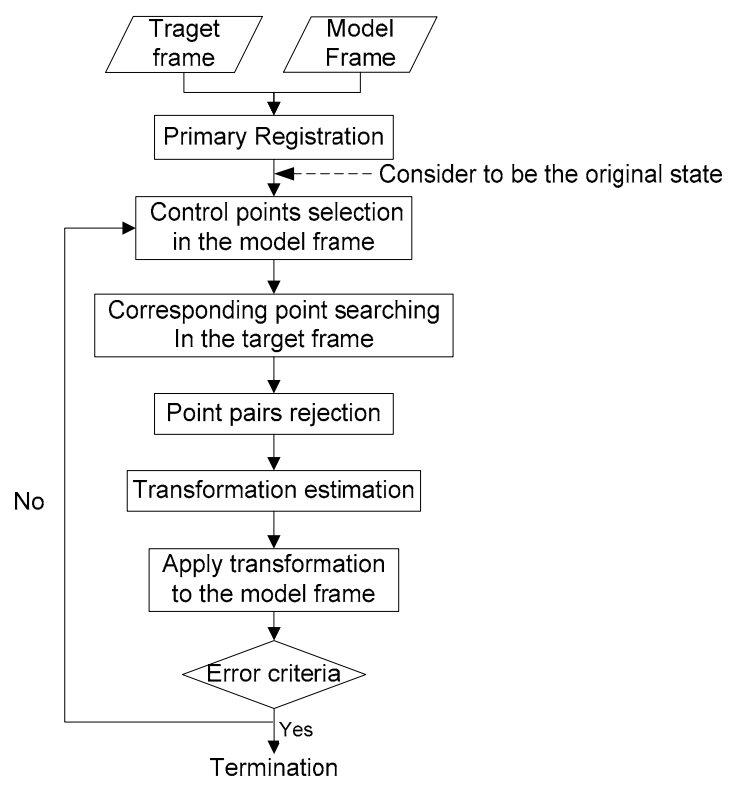

Proc. of SPIE Vol. $820082000 F-5$ 
Since the input of registration is increasing large owing to the improvement of the high resolution of camera, a common acceleration strategy is to select a subset of points in the model frame through sampling such as uniform sampling, random sampling, normal-space sampling and curvature-space sampling. The sampled points in the model frame are called control points, whose corresponding points are searched in the reference frame. Theoretically, the true corresponding pairs will be matched after several iterations. In practice, the corresponding pair for every control point is temporarily chosen through some closest distance strategy in each iteration. However, there are some corresponding point pairs whose distances are too much deviated from the average especially in the edge area of two partially overlapped frames. So it is necessary to reject some improper corresponding point pairs. The more accurate the correspondences are found, the much closer the transformation is estimated. In the following two subsections, the main contributions of my proposed ICP-based varient are explained in details.

\subsection{Correspondence selection by the aid of invariant features}

In the ICP pipeline, finding correspondence is one of the most important stages in the ICP pipeline. Currently, features from both surfaces can be used in assistance with correspondence searching. As I mentioned in the 2.2, the traditional ICP method only takes account of the spatial positions for correspondence searching. The corresponding points in the reference frame of the control points in the model frame are the Euclidean Closest Point or sometimes called Nearest Neighbor. In my proposed method, the principle curvatures are added into the weighted distance function for correspondence selection. Assume the spatial coordinate of a control point $q$ is denoted as $\mathbf{q}_{\mathbf{e}}$ and the feature coordinate is denoted as $\mathbf{q}_{\mathbf{f}}$, then we have

$$
\begin{aligned}
\mathbf{q}_{\mathrm{e}} & =\left(\mathrm{q}_{\mathrm{x}}, \mathrm{q}_{\mathrm{y}}, \mathrm{q}_{\mathrm{z}}\right) \in \mathrm{R}^{3} \\
\mathbf{q}_{\mathbf{f}} & =\left(\mathrm{q}_{k 1}, \mathrm{q}_{\mathrm{k} 2}\right) \in \mathrm{R}^{2} \\
\mathbf{q} & =\left(\mathbf{q}_{e}, \mathbf{q}_{\mathbf{f}}\right) \in \mathrm{R}^{3+2}
\end{aligned}
$$

where $\mathrm{q}_{k 1}, \mathrm{q}_{\mathrm{k} 2}$ are the principle curvatures of point q. Similarly, the corresponding point pair of q can be expressed as $\mathbf{p}$. The weighted combined of spatial position and feature distance between point $\mathrm{p}$ and q shall be denotes as:

$$
\mathbf{d}_{\alpha}(\mathbf{p}, \mathbf{q})=\mathbf{d}_{\mathrm{e}}\left(\mathbf{p}_{\mathrm{e}}, \mathbf{q}_{\mathrm{e}}\right)+\boldsymbol{\alpha}^{2} \mathbf{d}_{\mathrm{f}}\left(\mathbf{p}_{\mathrm{f}}, \mathbf{q}_{\mathrm{f}}\right)
$$

where

$$
\begin{aligned}
& \mathbf{d}_{\mathrm{e}}\left(\mathbf{p}_{\mathrm{e}}, \mathbf{q}_{\mathrm{e}}\right)=\left\|\mathbf{p}_{\mathrm{e}}-\mathbf{q}_{\mathrm{e}}\right\|^{2} \\
& \mathbf{d}_{\mathbf{f}}\left(\mathbf{p}_{\mathrm{f}}, \mathbf{q}_{\mathrm{f}}\right)=\left\|\mathbf{p}_{\mathrm{f}}-\mathbf{q}_{\mathrm{f}}\right\|^{2}
\end{aligned}
$$

and $\alpha$ is a constant in each iteration, which controls the contribution of the features. According to ${ }^{[9]}$, it proves that the estimation of error variance in each one spatial dimension (i.e. $\sigma_{z}^{2}$ ) due to misalignment approximates to the closest point distance $d_{e}$ expressed by the (11). Furthermore, the desired weight factor $\alpha$ after normalization by $\Sigma_{F}^{-1 / 2}$ is set to be the variance of one dimensional of positional error $\sigma_{z}^{2}$ in order to make the variance of feature error equals to the positional error. Therefore, $\alpha$ can be determined by $(13,14)$, the mean squared error represents the global estimate of $\mathrm{d}_{\mathrm{e}}^{2}$.

$$
\begin{gathered}
\sigma_{z}^{2}=\mathrm{E}\left(\mathrm{d}_{\mathrm{GTx}}^{2}\right)=\mathrm{E}\left(\mathrm{d}_{\mathrm{e}}^{2}\right) \approx \mathrm{d}_{\mathrm{e}}^{2} \\
\alpha^{2}=\sigma_{\mathrm{z}}^{2} \approx \mathrm{d}_{\mathrm{e}}^{2} \approx \operatorname{MSE}\left(\mathrm{d}_{\mathrm{e}}^{2}\right)
\end{gathered}
$$

In addition, K-d tree could be applied for searching acceleration.

\subsection{Pairs rejection using adaptive threshold of weighted distances}

If the target frame and the model frame are fully matching, every point in the target frame shall has a ground truth corresponding point in the model frame. It indicates that two frames measure the same region but the poses of the camera differs. The traditional ICP method only works in this situation. However, the target and the model frame are partially overlapped, which means the regions measured by the two frames are not identical in practice. In this case, the points of the model frame in the individual areas are often found the nearest edge points in the target frame to be their corresponding points, which are the spurious point pairs. Some constraints shall be imposed to remove them for more accurate estimation of transformation. The maximum tolerance of distance is exploited in my proposed method, inspiring from Zhang's method ${ }^{[10]}$. Instead of using all correspondence to estimate transformations, Zhang proposed an adaptive threshold for the screening the corresponding point pairs. This method makes the ICP method work for the partially 
overlapped point sets. The adaptive threshold (i.e. maximum tolerance of distance) is determined through the statistics of the distances of all corresponding point pairs. In my proposed method, the distance function is improved to be the weighted distance. That is, the objective of statistics is modified to be the weighted distance. The mean $\mu$ and standard deviation $\sigma$ of the weighted distances are given by (15).

$$
\mu=\frac{1}{N} \sum_{\mathrm{i}=1}^{\mathrm{N}} \mathrm{d}_{\alpha \mathrm{i}}, \sigma=\sqrt{\frac{1}{\mathrm{~N}} \sum_{\mathrm{i}=1}^{\mathrm{N}}\left(\mathrm{d}_{\alpha \mathrm{i}}-\mu\right)^{2}}
$$

Then, the threshold $\mathrm{D}_{\max }$ can be determined adaptively in each iteration as follows: if $\mu<D, \mathrm{D}_{\max }=\mu+3 \sigma$, the registration is quite good; if $D<\mu<3 D, \mathrm{D}_{\max }=\mu+2 \sigma$, the registration is acceptable; if $3 D<\mu<6 D, \mathrm{D}_{\max }=\mu+\sigma$, the registration is not too bad; if $\mu>6 D, D_{\max }=\zeta$; the registration is fairly bad. $\mathrm{D}$ is the resolution of the frames. $\zeta$ is set to the valley after the maximum peak of the weighted distance histogram.

\section{DATA, EXPERIMENTAL RESULTS AND DISCUSSIONS}

In the experiments, the proposed method is verified by using the artificial and real data respectively. The artificial data is assumed to be the ideal noise-free input for registration. The performance of the proposed method is evaluated in the aspect of the convergence and iteration times in the condition of full-matching case and partially overlapped case. The real data is produced by the self-developed FPP system.

\subsection{Artificial Data}

Firstly, a parametric surface is created by the following MATLAB code:

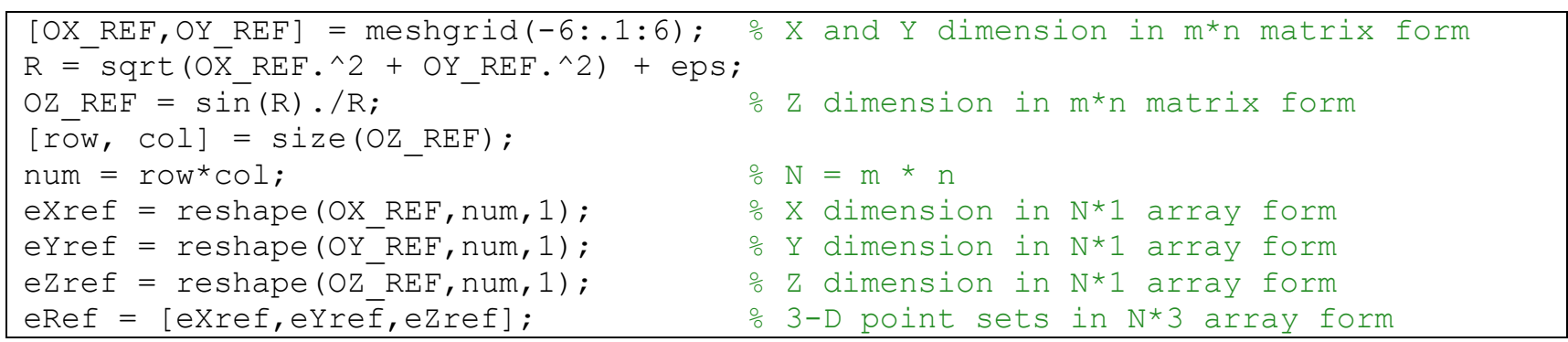

The parametric surface is generated twice. The first one maintains original and represents the target frame; while the other one rotated 5 degrees and translated 1.5, 0.5, 0.2 along $\mathrm{X}, \mathrm{Y}$ and $\mathrm{Z}$ axis respectively represents the model frame. In the Fig. 3, the red point set represents the target frame, the blue one represents the model frame, which is going to align to the target frame. The two point sets are simulated to be the ideal noise-free input data for the registration. In other words, Fig. 3 illustrates the original state of the full matching registration and the transformation between them is given as follows:

$$
\mathrm{T}=\left[\begin{array}{llll}
1.5 & 0.5 & 0.2
\end{array}\right], \mathrm{R}=\left[\begin{array}{ccc}
0.9924 & -0.868 & 0.0872 \\
0.0944 & 0.9917 & -0.0868 \\
-0.0789 & 0.0944 & 0.9924
\end{array}\right]
$$

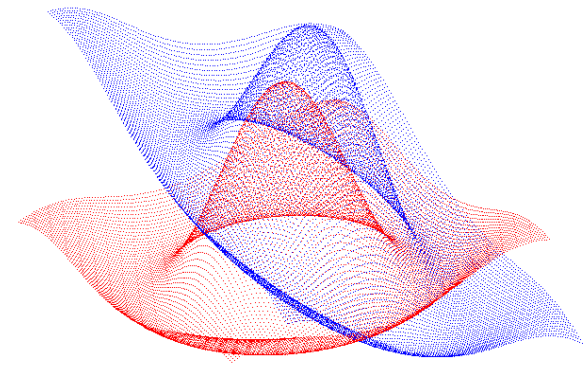

Fig. 3. Ideal 3-D complete (full matching) target frame, model frame and their original state 


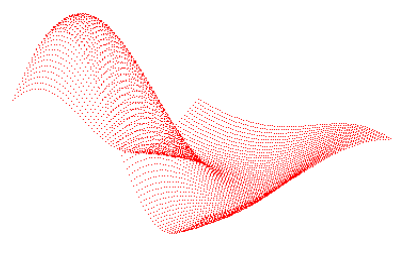

(a) Target frame

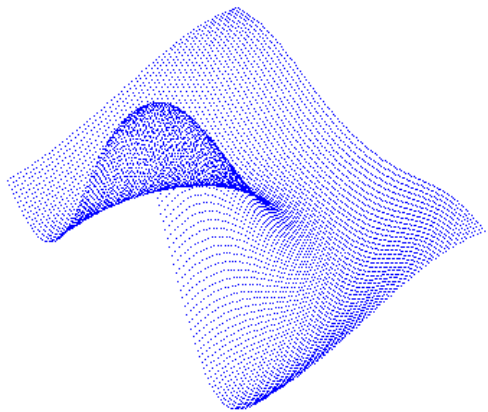

(b) Model frame

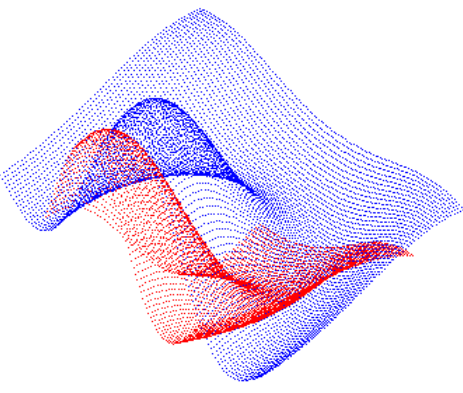

(c) Original state

Fig. 4. The ideal partially overlapped target frame, model frame and their original state

For the generation of partially overlapped frames, the target frame takes only $40 \%$ of the complete target frame, as shown in the Fig. 4(a). Fig. 4 (b) shows the model frame that is half portion of the complete model frame. Fig. 4(c) shows the original state for partially overlapped registration.

\subsection{Experiment I - Full matching registration using artificial data}

This experiment aims to implement the full matching registration by means of traditional ICP, Zhang's method and the proposed method respectively. The performances are evaluated through the precision of the transformation estimation and the convergence behavior.

First of all, the rotation and translation error are regarded as the precision of estimated results and are defined as (16) in ${ }^{[10]}$ :

$$
\mathrm{e}_{\mathrm{R}}=\frac{\|\mathrm{R}-\widehat{\mathrm{R}}\|}{\|\mathrm{R}\|} \times 100 \%, \mathrm{e}_{\mathrm{t}}=\frac{\|\mathrm{T}-\widehat{\mathrm{T}}\|}{\|\mathrm{T}\|} \times 100 \%
$$

where $\mathrm{R}$ and $\mathrm{T}$ are the given transformation, $\widehat{\mathrm{R}}$ and $\widehat{\mathrm{T}}$ are the estimated transformation. The transformations estimated by the three methods after 100 iterations and their precision are shown in the Table 1. Fig. 5 illustrates the aligned state after 100 iterations.

Table. 1 . The transformation estimations and precision after 100 iterations.

\begin{tabular}{|c|c|c|c|c|c|c|c|}
\hline & Measured Translation & Translation error & \multicolumn{4}{|c|}{ Measured Rotation } & Translation error \\
\hline Traditional ICP & $\widehat{\mathrm{T}}=\left[\begin{array}{llll}1.3673 & 0.4189 & 0.2882\end{array}\right]$ & $e_{T}=1.26 \%$ & $\widehat{\mathrm{R}}=$ & {$\left[\begin{array}{c}0.9933 \\
0.0753 \\
-0.0881\end{array}\right.$} & $\begin{array}{c}-0.829 \\
0.9928 \\
0.0862\end{array}$ & $\left.\begin{array}{c}0.0810 \\
-0.0929 \\
0.9924\end{array}\right]$ & $e_{R}=5.96 \%$ \\
\hline Zhang's ICP & $\widehat{\mathrm{T}}=\left[\begin{array}{llll}0.8135 & 0.2884 & 0.2981\end{array}\right]$ & $e_{T}=20.69 \%$ & $\widehat{\mathrm{R}}=$ & {$\left[\begin{array}{c}0.9953 \\
-0.0153 \\
0.0959\end{array}\right.$} & $\begin{array}{l}0.0234 \\
0.9962 \\
0.0837\end{array}$ & $\left.\begin{array}{c}-0.0943 \\
-0.0856 \\
0.9919\end{array}\right]$ & $e_{R}=4.97 \%$ \\
\hline Proposed ICP & $\widehat{\mathrm{T}}=\left[\begin{array}{llll}1.2824 & 0.4299 & 0.2958\end{array}\right]$ & $e_{T}=2.42 \%$ & $\widehat{\mathrm{R}}=$ & $\begin{array}{c}0.9959 \\
-0.0196 \\
0.0881\end{array}$ & $\begin{array}{c}0.0272 \\
0.9959 \\
-0.0865\end{array}$ & $\left.\begin{array}{c}-0.0860 \\
0.0885 \\
0.9924\end{array}\right]$ & $e_{R}=4.91 \%$ \\
\hline
\end{tabular}




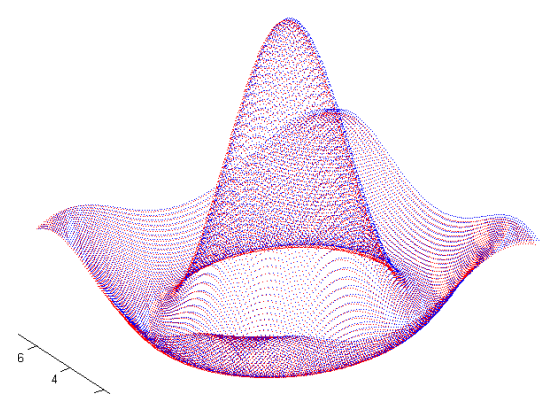

(a) Traditional method

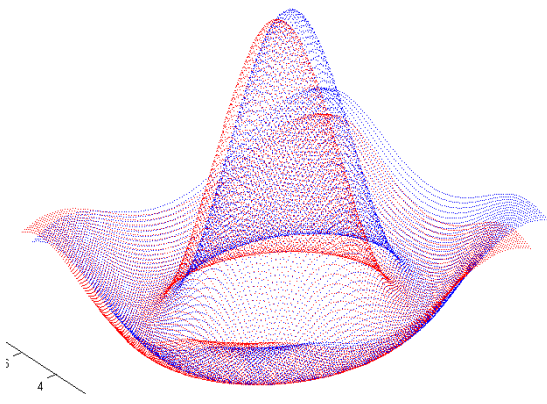

(b) Zhang's method

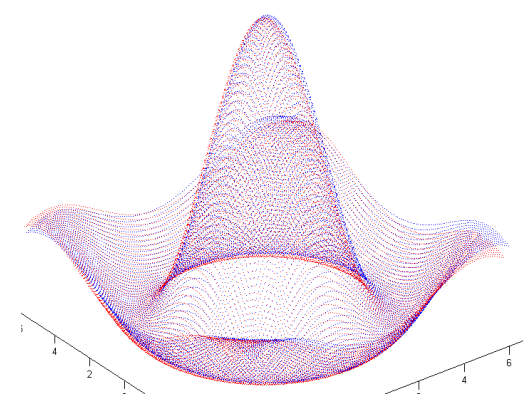

(c) Proposed method

Fig. 5. The aligned state after 100 iterations.

By comparison, the accuracy of traditional method and the proposed method is much higher than the Zhang's method. This is because that the number of corresponding point pairs for transformation estimation used by the traditional method is much more than the other two methods. It is known that the more point pairs there are, the higher accurate the results are, but the more the consumption time takes. While both Zhang's method and the proposed method use less point pairs for estimation by applying a threshold, which leads to the accuracy reduction. However, the precision of the proposed method is all close to that of the traditional method, which owe to the advanced searching strategy.

Secondly, the convergence behaviors of the three methods are evaluated. The comparisons are based on the mean square error of distances between the corresponding point pairs. The MSE of the three methods are compared in the Fig. 6.

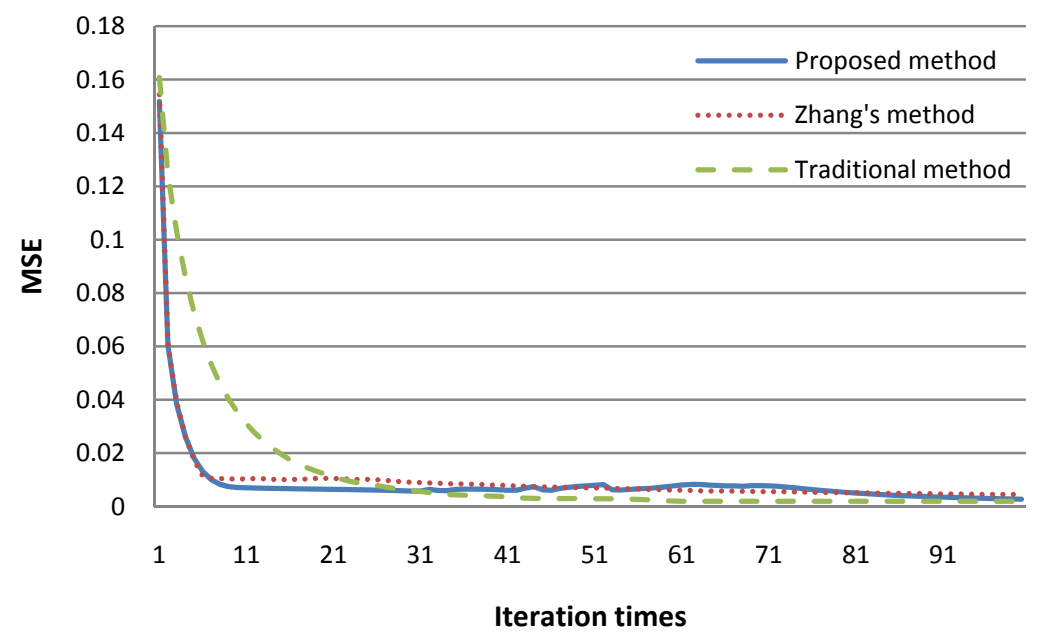

Fig. 6. The convergence behavior of the full matching registration in 100 iterations.

It is seen that Zhang's method and the proposed method converge much faster than the traditional method. It proves that the selection strategy has a massive impact on the convergence.

\subsection{Experiment II - Partially overlapped registration using artificial data}

In this experiment, the performances of the partially overlapped registration are only evaluated by Zhang's method and the proposed method, due to that the traditional method only works in the full-matching case. The partially overlapped target frame, model frame and their original state are shown in the Fig. 4 (a) (b) respectively. Fig. 7 illustrates the aligned results. 


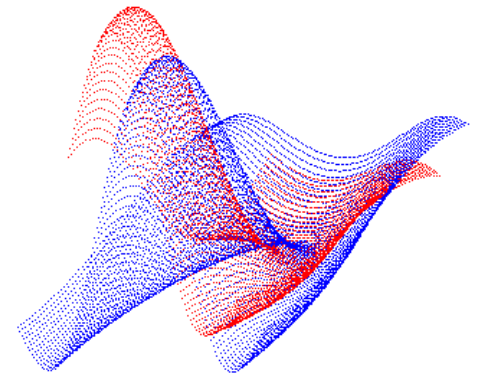

(a) Zhang's method

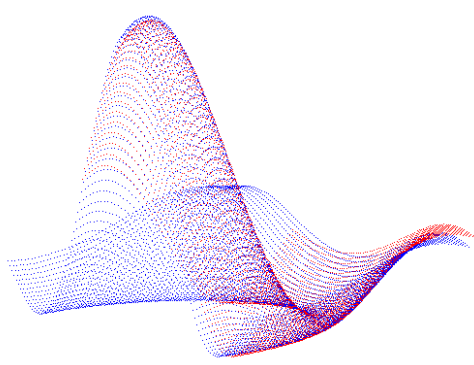

(b) The proposed method

Fig. 7. The aligned state after 100 iterations

Table. 2. The transformation estimations and precision after 100 iterations

\begin{tabular}{|c|c|c|c|c|c|}
\hline & Measured Translation & Translation error & Measured Rotation & Rotation error \\
\hline \multirow{2}{*}{ Zhang's ICP } & $\widehat{\mathrm{T}}=\left[\begin{array}{llll}0.5566 & 0.09610 .5450\end{array}\right]$ & $e_{T}=46.15 \%$ & $\widehat{\mathrm{R}}=\left[\begin{array}{ccc}0.9897 & 0.1015 & -0.1006 \\
-0.0979 & 0.9943 & -0.0432 \\
0.1044 & 0.0330 & 0.9940\end{array}\right]$ & $e_{R}=5.81 \%$ \\
\hline \multirow{2}{*}{ Proposed ICP } & $\widehat{\mathrm{T}}=\left[\begin{array}{llll}1.5498 & 0.3632 & 0.2681\end{array}\right]$ & $e_{T}=1.02 \%$ & $\widehat{\mathrm{R}}=\left[\begin{array}{ccc}0.9822 & 0.1749 & -0.0684 \\
-0.1673 & 0.9804 & 0.1039 \\
-0.0852 & 0.0906 & 0.9922\end{array}\right]$ & $e_{R}=8.63 \%$ \\
\hline
\end{tabular}

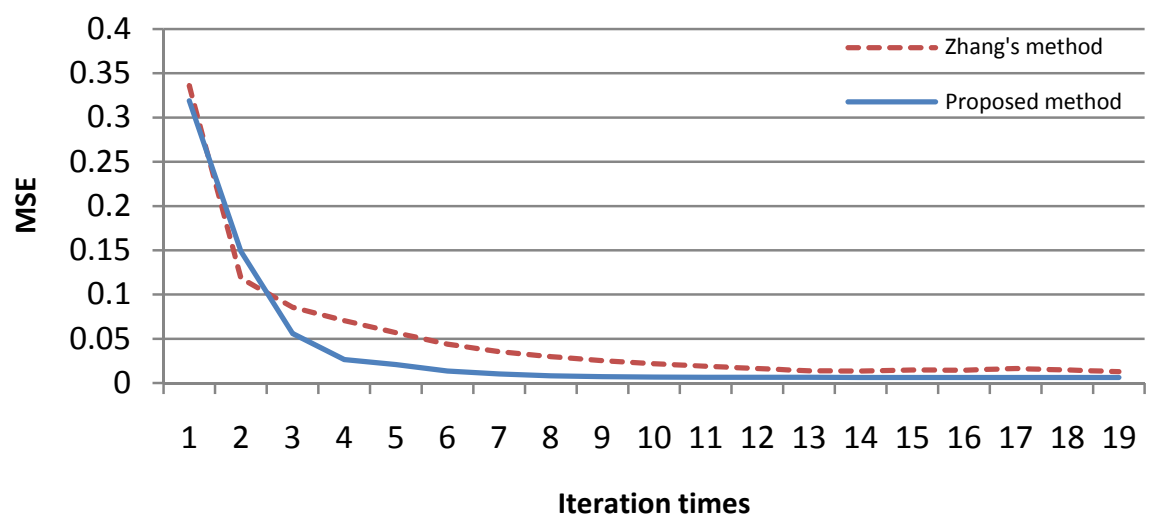

Fig. 8. The convergence behavior of the partially overlapped registration in the first 20 iterations

It is clearly seen that the precision of the translation estimated by the proposed method is much higher than the estimation of Zhang's method. By comparison, the rotation error estimated by the two methods varies slightly. In general, the performance of the proposed method is better than Zhang's method, as illustrated in the Fig. 7. In the section 3.2, the advance of the proposed method is explained as that it could find more accurate corresponding point pairs during each iteration. In other words, it shall estimate more accurate and converges faster. Fig. 8 verifies the expectation.

\subsection{Experiment III - Partially overlapped registration using real data}

The self-developed FPP system is composed of a DuncanTech MS3100 3-CCD camera with Nikon AF-S DX NIKKOR 16-85mm f/3.5-5.6 ED VR lens, a HITACHI CP-X260 Multimedia LCD projector and a PC with Intel Core i7 860 CPU @ $2.80 \mathrm{GHz}$ and 2.96GB of RAM. The real data is produced by this FPP system, as shown in the Fig. 9 (a) and (b). The transformation between the target frame and the model frame is:

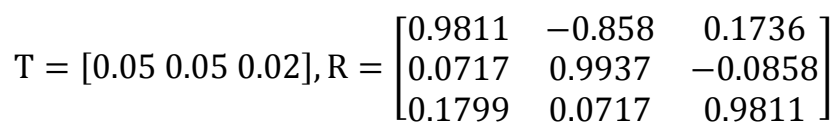




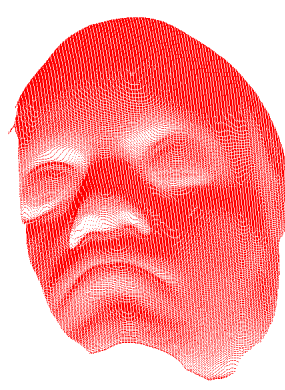

(a) Target frame

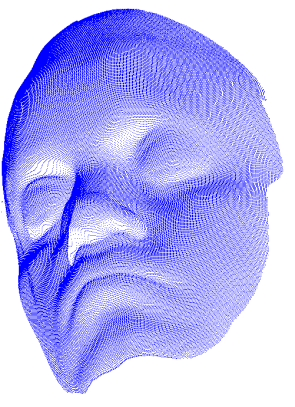

(b) Model frame

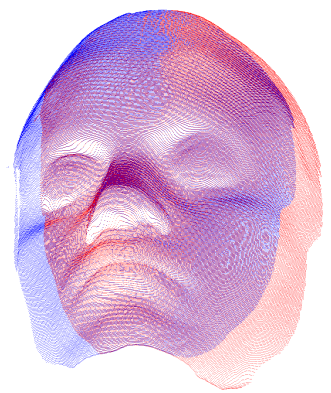

(c) Aligned result

Fig. 9. The partially overlapped registration using real data

Fig. 9 (c) shows the registration of real data by the proposed method. The MSE converges to a zero at the $31^{\text {st }}$ iteration. The estimated transformation at the $31^{\text {st }}$ iteration is:

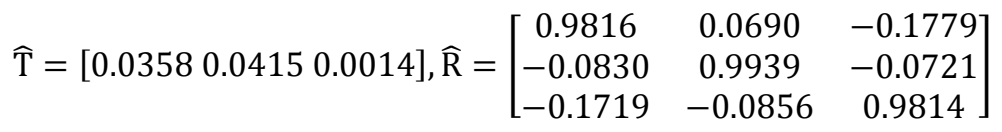

and the precision achieves to $11.45 \%$ and $11.50 \%$ respectively.

\section{CONCLUSIONS}

This paper presents a pair-wise registration method to align partially overlapped range images generated by FPP system. This method is based on the classic ICP method but has some extensions in accordance with the data generated by self-developed FPP system. A series of experiments are carried out and the experimental results show that the proposed method can increase the speed of convergence and the accuracy of estimation.

\section{REFERENCES}

[1] Bernardini, F. and Rushmeier, H., "The 3D Model Acquisition Pipeline," Comput. Graphics Forum, 21(2), 149-172 (2002).

[2] Hu, Y., "Research on Digital Projection based Three-dimensional Fringe Pattern Profilometry," Degree of Doctor of Philosophy in Engineering, Huazhong University of Science and Technology, Wuhan, 2006.

[3] Gagnon, H., Soucy, M., Bergevin, R., and Laurendeau, D., "Registration of multiple range views for automatic 3-D model building," Proc. IEEE CVPR, 581-586(1994).

[4] Besl, P. J. and McKay, H. D., "A method for registration of 3-D shapes," IEEE Trans. Pattern Anal. Mach. Intell., 14(2), 239-256 (1992).

[5] Arun, K. S., Huang, T. S., and Blostein, S. D., "Least-Squares Fitting of Two 3-D Point Sets," IEEE Trans. Pattern Anal. Mach. Intell., 9(5), 698-700 (1987).

[6] Horn, B. K. P., "Closed-form solution of absolute orientation using unit quaternions," J. Opt. Soc. Am. A:, 4(4), 629-642 (1987).

[7] Walker, M. W., Shao, L., and Volz, R. A., "Estimating 3-D location parameters using dual number quaternions," CVGIP: Image Understanding, 54(3), 358-367 (1991).

[8] Rusinkiewicz, S. and Levoy, M., "Efficient variants of the ICP algorithm," Proc. The third International Conference on 3-D Digital Imaging and Modeling, 145-152(2001).

[9] Sharp, G. C., Lee, S. W., and Wehe, D. K., "ICP registration using invariant features," IEEE Trans. Pattern Anal. Mach. Intell., 24(1), 90-102 (2002).

[10] Zhang, Z., "Iterative point matching for registration of free-form curves and surfaces," Int. J. Comput. Vision, 13(2), 119-152 (1994).

[11] Zinsser, T., Schmidt, J., and Niemann, H., "A refined ICP algorithm for robust 3-D correspondence estimation," Proc. International Conference on Image Processing II-695-8 vol.3(2003).

[12] Trucco, E., Fusiello, A., and Roberto, V., "Robust motion and correspondence of noisy 3-D point sets with missing data," Pattern Recognit. Lett., 20(9), 889-898 (1999). 
Masuda, T. and Yokoya, N., "A Robust Method for Registration and Segmentation of Multiple Range Images," Comput. Vision Image Understanding, 61(3), 295-307 (1995).

[14] Chua, C. S. and Jarvis, R., "Point Signatures: A New Representation for 3D Object Recognition," Int. J. Comput. Vision, 25(1), 63-85 (1997).

Johnson, A. E. and Hebert, M., "Surface registration by matching oriented points," Proc. International Conference on Recent Advances in 3-D Digital Imaging and Modeling, 121-128(1997). Feldmar, J. and Ayache, N., "Locally affine registration of free-form surfaces," Proc. IEEE CVPR, 496501(1994).

Zhang, S. and Yau, S.-T., "Generic nonsinusoidal phase error correction for three-dimensional shape measurement using a digital video projector," Appl. Opt., 46(1), 36-43 (2007). Besl, P. J. and Jain, R. C., "Invariant surface characteristics for 3D object recognition in range images," Computer Vision, Graphics, and Image Processing, 33(1), 33-80 (1986). Feldmar, J. and Ayache, N., "Rigid, affine and locally affine registration of free-form surfaces," Int. J. Comput. Vision, 18(2), 99-119 (1996).

Gelfand, N., Mitra, N. J., Guibas, L. J., and Pottmann, H., "Robust global registration," Proc. The third Eurographics symposium on Geometry processing, 197(2005).

[21] Temerinac-Ott, M., Keuper, M., and Burkhardt, H., "Evaluation of a New Point Clouds Registration Method Based on Group Averaging Features," Proc. the 20th International Conference on Pattern Recognition (ICPR), 2452-2455(2010). 